\title{
ANÁLISIS ECONÓMICO DE LA ADMINISTRACIÓN DE JUSTICIA: ¿LA JUSTICIA COMO BIEN PÚBLICO O PRIVADO?
}

\author{
ECONOMIC ANALYSIS OF JUSTICE ADMINISTRATION: \\ ¿JUSTICE AS A PUBLIC OR PRIVATE GOOD
}

\author{
Raúl NúNEZ OJeda ${ }^{1}$ \\ Nicolás Carrasco Delgado ${ }^{2}$
}

\begin{abstract}
RESUMEN: Este trabajo se enmarca en la disputa del concepto de justicia como bien público. Utilizando herramientas de Análisis Económico del Derecho, se cuestionarán las tesis tradicionales existentes en Chile que consideran la administración de justicia como bien privado. En el trabajo se reafirma el carácter de bien público de la justicia, debiendo para ello descartar los presupuestos de las tesis tradicionales que abogan por su privatización. Se propone, una nueva forma de entender la labor jurisdiccional, considerando la eficiencia, como criterio de decisión judicial. De esa forma se obtendrán externalidades públicas positivas que justifiquen la inversión estatal en justicia.
\end{abstract}

Palabras clave: Derecho Procesal, Análisis Económico del Derecho, Acceso a la justicia, Precedentes.

ABSTRACT: This paper focuses in the debate of a concept of justice conceived as a public good. By using tools of the Law and Economics, the traditional theories existing in Chile that considers the administration of justice as a private good, will be questioned. In the following pages, the essential condition of justice as a public good is argued, in order to dismiss the arguments offered by traditional theories that suggest the privatization of justice. The article proposes a new way to understand the jurisdictional function, considering efficiency as reasoning criteria of judicial decisions. Thus, is possible to obtain positive public externalities that justify the state investment in justice.

Key words: Procedural law, Law and Economics, Access to Justice, Precedents.

\section{NOTAS INTRODUCTORIAS 3}

Según cierta literatura nacional sobre análisis económico del derecho procesal ${ }^{4}$, los servicios de justicia (problema desde la perspectiva de la oferta) prestados por los tribunales

${ }^{1}$ Licenciado en Ciencias Jurídicas por la Universidad de Valparaíso (Chile); Doctor en Derecho por la Universidad Pompeu Fabra de Barcelona (España); Profesor Asociado de Derecho Procesal en la Pontificia Universidad Católica de Valparaíso (Chile). Dirección postal: Avenida Brasil 2950, Valparaíso, Chile. Correo electrónico: raul.nunez@ucv.cl

${ }^{2}$ Licenciado en Ciencias Jurídicas y Sociales por la Universidad de Chile. Magíster con mención en Derecho Económico de la Facultad de Derecho de la Universidad de Chile. Profesor de Derecho Procesal Universidad de Chile. Dirección postal: Pío Nono 1, Santiago, Chile. Correo electrónico: ncarrascod@gmail.com

3 Se agradece la valiosa colaboración prestada por nuestra ayudante de la Universidad de Chile Fernanda Skewes, por sus aportes y ayuda en la redacción de este artículo.

${ }^{4}$ Merr (2006) y Correa et al. (2000). 
que dependen del Poder Judicial, y que por tanto, son financiados con cargo a rentas generales, no deben constituir bienes públicos, sino que esencialmente privados, si es que constatamos que no permiten alcanzar externalidades positivas públicas (precedentes).

En efecto, tales servicios al estar financiado por el Estado, y por tanto, con recursos públicos, se esperaría que produjesen externalidades positivas públicas (precedente), y para el caso que ello no se cumpliera, debería optarse por mecanismos de resolución de disputas de carácter alternativos (por ejemplo, arbitraje, mediación, etc.), financiados fundamentalmente por quienes acceden a dichos mecanismos de resolución de conflicto, de forma de dar cuenta de la verdadera naturaleza de los servicios de justicia (bien privado).

Adicionalmente, y ahora desde la perspectiva de la demanda, en aquellos casos en los cuales, el costo privado del litigio se divorcia del costo público del mismo, se debería hacer uso de instrumentos tendientes a hacer coincidir tales costos, por medio de tasas judiciales.

Se expresará en este trabajo, que no se comparte la mencionada visión de los servicios de justicia, tanto desde la perspectiva de la oferta (entender los servicios como bien público o privado), como desde la perspectiva de la demanda (fijación de tasas judiciales a ser pagadas por los usuarios de servicios de justicia). Ello en atención a que, desde la perspectiva de la oferta, la existencia de un servicio financiado por el Estado que no genera externalidades positivas públicas no trae consigo como solución óptima transformar el sistema total o parcialmente en un bien privado. Y además, porque desde la perspectiva de la demanda de servicios de justicia, el establecimiento de desincentivos a la litigación no óptima, por medio de la fijación de tasas para hacer uso del derecho a accionar, supone una intervención regulatoria costosa y difícilmente aplicable, en términos tales, que la supuesta solución significará mayores niveles de ineficiencia que las problemáticas actualmente vigentes.

En lo que sigue de este trabajo, denominaremos como tesis tradicional, a aquellos postulados que sostienen una posición crítica al financiamiento estatal de servicios de justicia que no generan externalidades positivas públicas, y que exigen el establecimiento de tasas judiciales para incentivar comportamientos de litigación óptimos. Los esquemas básicos de la propuesta de la tesis tradicional serán tratados en el acápite II de este trabajo. Por su parte, en el acápite III, se manifestarán las razones que hacen improcedente la tesis tradicional. Para finalmente, en el acápite IV, establecer las conclusiones respectivas.

El marco teórico en el que se enfoca este texto, se encuentra en los postulados del Análisis Económico del Derecho, al que recurriremos en sus concepciones más básicas.

\section{EL ESQUEMA BÁSICO DE LA TESIS TRADICIONAL}

Dentro de lo que denominamos tesis tradicional encontramos dos trabajos clásicos sobre este tema, y que corresponden al artículo publicado por Jorge Correa, Carlos Peña y Juan Enrique Vargas, denominado: “¿Es la justicia un bien público?”, y el trabajo de Rafael Mery Nieto, titulado: "Una aproximación Teórica y Empírica a la Litigación Civil en Chile"6.

\footnotetext{
Correa et al. (2000).

6 Mery (2006) p. 38.
} 
En primer lugar, desde la perspectiva de la oferta, los autores mencionados cuestionan que los servicios de justicia como los entendemos actualmente, se configuren como un bien público, esto es, como: "un servicio público destinado a proveer dicho servicio, a cargo de funcionarios remunerados por el Estado, financiado con cargo a rentas generales y sin barreras aparentes de acceso como lo sería, por ejemplo, un sistema de precios"

Más bien, para los mencionados autores, la composición de la justicia civil y comercial, determinan que se trata de un bien privado. Al respecto, su diagnóstico es el siguiente:

"Un examen de la justicia civil y comercial - de la composición del litigio y de su comportamiento- pone de manifiesto que en esas áreas la justicia no constituye un bien público. No es verdad, desde luego, que la incorporación de un nuevo litigante carezca de costos, que no exista rivalidad por acceder al aparato de justicia o que no sea posible excluir a un consumidor. Ninguno de esos rasgos - propios de un bien público- los presenta la justicia civil y comercial. Ese tipo de justicia constituye un bien privado, cuyos beneficios -aunque no todos sus costos- los internaliza predominantemente los litigantes. Al proveerse ese tipo de justicia como si fuera un bien público se produce un resultado socialmente ineficiente: como los litigantes no pagan todos los costos asociados a ese bien, se acrecienta su tasa de bienestar asociada al litigio. Los sujetos tenderán, entonces, a litigar más, incluso más allá de lo que resultaría eficiente desde el punto de vista del bienestar social. Aunque los costos asociados al litigio sean superiores al beneficio que con él se obtiene, de todas suertes los sujetos tenderán a demandar. Dicho en términos técnicos, existe una divergencia entre los costos sociales y privados del litigio"s.

Como diremos posteriormente, constataremos que el análisis que se realiza en el párrafo recién transcrito no ayuda a clarificar ciertos conceptos relevantes.

Ahora bien, los autores indicados al efectuar un análisis para descartar la existencia de un bien público desde la perspectiva de la oferta, terminan erradamente en nuestro entender, derivando una problemática relativa a la demanda de servicios de justicia9 ${ }^{9}$ En otras palabras, metodológicamente la forma que realizan los autores indicados para el tratamiento del problema es poco clara, ya que, no identifican las problemáticas asociada a los servicios de justicia desde la perspectiva de la oferta, sino que de ella, concluyen ciertas consideraciones referentes a problemáticas desde la perspectiva de la demanda (litigación privada sobre el óptimo social). Ese proceder metodológico tiende a repetirse en los trabajos mencionados ${ }^{10}$.

\footnotetext{
CORREA et al. (2000) p. 391.

${ }^{8}$ Correa et al. (2000) pp. 391-392.

9 "Al proveerse ese tipo de justicia como si fuera un bien público se produce un resultado socialmente ineficiente: como los litigantes no pagan todos los costos asociados a ese bien, se acrecienta su tasa de bienestar asociada al litigio" Correa et al. (2000) p. 391.

${ }^{10}$ Por ejemplo, Vargas, Peña y Correa, señalan: "Solo poniendo un precio a la justicia se evitará la litigación ineficiente y se salvará al sistema de su colapso. Ello, por supuesto, en nada se opone al establecimiento de subsidios directos en favor de las personas que carezcan de los recursos necesarios para proporcionarse este bien. De esta forma se podrá poner fin al carácter regresivo de la inversión en justicia" (CORREA et al. (2000) p. 401). Tales autores explicaron que el carácter de regresivo de los gastos de justicia se debía a que la inversión de justicia no iba aparejada de un efecto disuasivo asociado a las externalidades que se producían por el sistema de administración de justicia. La regresividad del gasto, es por lo tanto, un problema desde la perspectiva de la oferta. Sin embargo, el esta-
} 
Finalmente, como un corolario de lo señalado, Vargas, Peña y Correa, expresan que: "la provisión de justicia es óptima cuando: (a) todos están protegidos por el efecto disuasivo o represivo y (b) cuando el Estado subsidia la demanda en una magnitud equivalente a las externalidades positivas que genera quien recurre al sistema"11.

Veremos en el capítulo siguiente que el efecto disuasivo en un sistema de resolución de conflicto como el que conocemos (adjudicación en base al caso concreto), es un imposible en términos absolutos, y en lo que es susceptible de mejora, requiere de reformas sobre la casación y el régimen recursivo que hacen demasiado costosa su implementación. Respecto de la posibilidad de subsidiar el acceso a la justicia, surge la problemática no menos inalcanzable de calcular el monto óptimo relativo a que la cuantía del subsidio de la demanda sea equivalente a las externalidades positivas de quien concurra. De esta forma, los supuestos de óptimos de oferta de la tesis tradicional resultan inalcanzables.

En lo que respecta a la demanda de servicios judiciales, el esquema básico supone que un potencial litigante decidirá acudir a la justicia si y solo si:

$$
P(W-M-C l+M)+(1-P)(W-M-C l) \geq W-M
$$

Donde:

$\mathrm{P}$ : probabilidad de que el demandante gane el caso;

W : renta bruta inicial del demandante;

$\mathrm{Cl}$ : costos monetarios del juicio para el demandante (abogados, tiempo, etc.).

M : cantidad monetaria reclamada (cuantía del juicio).

Por lo tanto, según Rafael Mery -siguiendo a Schavell- de acuerdo a la expresión anterior, el individuo decidirá acudir a la justicia única y exclusivamente si la ganancia marginal neta de acudir a la justicia es positiva. O sea, si:

$$
p \geq \frac{C l}{M}=\mathrm{p}^{* 12}
$$

Donde $\left(\mathrm{p}^{*}\right)$, sería el "nivel crítico de probabilidad para acceder a la justicia", esto es, según Mery, aquel nivel que determina el límite en base al cual el potencial litigante decidirá acudir o no a la justicia.

blecimiento de desincentivos para demandar - por medio del establecimiento de tasas-, tienden a solucionar el problema desde la perspectiva de la demanda. Con ello se demuestra que los autores tratan metodológicamente ambas dificultades como un solo problema, sin las necesarias prevenciones de que se trata de asuntos diferentes.

11 Vargas, et al. (2001) p. 21.

12 En este sentido, el desarrollo de la ecuación es la siguiente:

$$
\begin{gathered}
P(W-M-C l+M)+(1-P)(W-M-C l) \geq W-M \\
P(W-C l)+(1-P)(W-M-C l) \geq W-M \\
P W-P C l+[(W-M-C l)(-p W+P M+P C l) \geq W-M \\
(P M) \geq C l \\
P \geq \frac{C l}{M}
\end{gathered}
$$


Siguiendo con el análisis que realiza Mery, dicho autor indica que si los costos monetarios del juicio para el demandante son mayores que la cantidad reclamada $(\mathrm{Cl}>\mathrm{M})$, entonces, nunca recurrirá a los servicios de justicia, de forma que es condición necesaria para que el individuo decida acudir a la justicia que $(\mathrm{Cl}<\mathrm{M})$.

A continuación, tal autor expresa, que $\mathrm{M}>\mathrm{Cl}$, entonces, $\mathrm{W}>\mathrm{Cl}$, toda vez que, si $\mathrm{W} \geq \mathrm{M}$, resulta razonable pensar que un individuo nunca perderá -como consecuencia del juicio-, más renta de la que inicialmente tenía. De ello se sigue que: (i) Si el demandante gana el juicio su renta será siempre positiva (W - Cl), y (ii) Si pierde el juicio, su renta podrá ser negativa o positiva, dependiendo de los valores involucrados ( $\mathrm{W}-\mathrm{M}-\mathrm{Cl}$ ).

La conclusión (ii) anterior, puede originar que siendo eficiente que exista un litigio $(\mathrm{M}>\mathrm{Cl})$, el mismo no llegue a iniciarse, toda vez que, la posibilidad del demandante de ser pernicioso en el resultado del juicio determine una renta negativa futura, que lo desincentive a litigar ${ }^{13}$. Frente a ello, surgen alternativas que permitan -desde las políticas públicas- enfrentar dicha problemática.

$\mathrm{Al}$ respecto, Mery, señala que las alternativas serían establecer un sistema de justicia gratuita en beneficio de aquellos que no tienen posibilidad de acceder a justicia, o bien, diseñar un sistema de costas que favorezca a la parte más débil económicamente.

En este trabajo no abordaremos la utilidad de este último mecanismo, sin perjuicio de recomendar lecturas básicas sobre el tema de la eficiencia de los sistemas de $\operatorname{costas}^{14}$, y asimismo, de hacer presente que el legislador puede establecer una regulación de óptimo de sistemas de costas con el objetivo de incentivar que el interés privado de concurrir por servicios de justicia, se adecue al óptimo social de litigación, eliminando demandas frívolas, aun cuando se aumenten los costos de demandas legítimas. Lo relevante es que para que funcione un sistema de costas, en base a criterios de eficiencia, se requiere no solamente claridad normativa, sino que adicionalmente el establecimiento de incentivos adecuados para motivar una cantidad de litigación consistente con el nivel de litigación socialmente óptima ${ }^{15}$.

En lo que se refiere al sistema de justicia gratuita, Mery, señala que una política en tal sentido, supondría que los costos monetarios del litigio para el demandante pasarían a no existir $(\mathrm{Cl}=0)$, y que por tanto, se relajarían los supuestos de la fórmula:

$$
p \geq \frac{C l}{M}=\mathrm{p}^{*}
$$

Lo anterior conduciría, evidentemente, a un nivel de litigación mayor del óptimo. Toda vez que aquellos litigantes que antes del establecimiento de un sistema de justicia

13 Dicha decisión sería apropiada si estamos frente a un litigante adverso al riesgo, y quizás sería aplicable también, para un litigante neutral al riesgo, mas no para un litigante propenso al riesgo.

14 Huerta (2012) y Hughes y Snyder (1995).

15 En este sentido, es divergente con el interés socialmente óptimo de litigación la existencia de interés particulares no consistentes con la finalidad de obtener una adecuada decisión del conflicto -sino que motivada, por el contrario, con la búsqueda de la venganza privada, o simplemente por motivos de frivolidad-. Por lo mismo, un sistema de costas eficiente puede incentivar la disminución de demandas frívolas, con lo cual, se conseguirá un mayor nivel de litigación socialmente óptima. En cuanto a la posibilidad de que una regulación de costas influya en las decisiones de demanda de justicia de posibles litigantes, ver: HuerTa (2012), pp. 112-113. 
gratuita, poseían restricciones presupuestarias $(\mathrm{Cl}>0)$, que hacían inviable el inicio del proceso, ahora podrán demandar al no existir dicha restricción. Al respecto, cabe agregar que una política en dicho sentido se encuentra afecta a la desventaja de establecer incentivos perversos en beneficio de quienes no se encuentran afectados por la restricción presupuestaria que estamos refiriendo $-\mathrm{y}$ que por tanto, no serían destinatarios naturales de una política de acceso gratuito a justicia-, pero que se harían pasar por aquellos que sí son destinatarios de la mencionada regulación (free riders).

Dada dicha constatación, es que Mery plantea soluciones contradictorias, ya que un primer momento señala que lo deseable sería establecer programas distributivos que garanticen que el nivel de renta inicial (W) sea lo suficientemente grande, como para que todos los individuos de la sociedad y potenciales litigantes, posean capacidad económica suficiente para generar renta positiva cualquiera sea el resultado del proceso ${ }^{16}$. Sin embargo, posteriormente, en oposición a dicha idea, señala la necesidad de subsidiar la litigación de quienes, no poseen suficientes recursos para acceder a la justicia, argumentando acerca de la justificación constitucional de dicho subsidio ${ }^{17}$.

Resulta evidente que la primera solución constituye un imposible desde las políticas públicas, porque es impensado que se quiera pretender que para solucionar un estado de litigación no óptima se busque aumentar las rentas generales de todos los habitantes de un país. Por lo mismo, se omitirá cualquier análisis al respecto. En relación con la segunda solución, no se establece por el autor, ni por ninguno de quienes sostienen la denominada tesis tradicional, la forma de cálculo, y características del subsidio a la litigación de quienes poseerían una capacidad económica que no le permitiera litigar. Lo anterior, incluso más allá de las dificultades para poder detectar, establecer y calcular aquellos elementos que harían posible el establecimiento de un subsidio óptimo.

Vinculado con ello, y buscando el establecimiento de sistemas de litigación que conduzcan a mejoras de la justicia civil y comercial, es que los exponentes de la tesis tradicional, señalan que la manera de hacer coincidir el interés privado en litigación, con el nivel óptimo de judicialización social, se alcanza por medio del establecimiento de tasas judiciales.

Lo anterior conduce a que Mery sostenga que: "resulta pertinente aconsejar que el Estado no provea íntegramente el bien, sino que cobre tasas a los litigantes (...)"18; “(...) aparece como necesario el establecimiento de tasas judiciales, lo que se justifica ya que en nuestro sistema, al no contar con estas tasas, el litigante solo cubre sus costos privados, y con cargo a rentas generales se financia el sistema judicial en su conjunto. El establecimiento de algún sistema de tasas que permita internalizar los costos del litigio, funciona-

\footnotetext{
16 "Así, la justicia gratuita traería aparejada ciertas ineficiencias que no serían del todo deseables. La opción, en cambio, puede ser a través de la implementación de programas distributivos que garanticen que el nivel de renta bruta inicial sea suficientemente grande, tal que W-M-Cl $\geq 0$, para todos los individuos de la sociedad y potenciales litigantes, manteniendo de esa manera la condición (2)” MerY (2006) p. 5.

17 "El establecimiento de un subsidio de esta naturaleza se justifica ya no solo por ser un derecho garantizado por nuestra Constitución Política, sino además porque así se evita que el potencial litigante que, teniendo razones para litigar no pueda cubrir sus costos, recurra a mecanismos más baratos, pero socialmente más perjudiciales” Mery (2006) p. 33.

18 Correa et al. (2000) p. 400.
} 
ría como una barrera de entrada a la litigación y así se lograría aproximar los costos sociales y los costos privados de la misma"19.

Sin embargo, los autores de la denominada tesis tradicional expresan una política judicial deseable, pero no administrable ${ }^{20}$. En efecto, si bien Mery, no avanza en la definición de qué sistema de tasas considera deseable, ello si es afrontado por Correa, Peña y Vargas, al expresar que la tasa del litigante debe "(...) cubrir el importe del servicio proporcionado menos el monto proporcional de las externalidades generadas" 21 .

Como veremos posteriormente (acápite 3.2) la solución mencionada resulta más costosa de implementar, y por tanto, constituiría un peor escenario que el sistema actual. En lo que sí podría existir consenso es en relación con la factibilidad de hacer procedente procedimientos más adversariales de resolución de conflictos, como sería por ejemplo, ampliar los supuestos de procedencia de los arbitrajes u otras formas alternativas de resolución de conflictos. En este sentido, por ejemplo, la reforma a la legislación de quiebras establece una regulación no solamente sobre procedimientos de liquidación, sino que también, de reorganización ${ }^{22}$.

Finalmente, es importante indicar que los modelos de litigación diferencian el litigante en atención al riesgo que representa (adverso, neutral o propenso al riesgo ${ }^{23}$ ). Sin embargo, nos centraremos exclusivamente en los modelos o formulaciones respecto de los litigantes adversos al riesgo, toda vez que, por un asunto de administravidad de las reglas no es posible que el legislador establezca una regulación procesal para diferentes modelos de litigantes en atención a cuál sea su vínculo con el riesgo.

\footnotetext{
19 Mery (2006) p. 34.
}

20 En este sentido, la administratibilidad de las reglas legales constituye un aspecto trascedente para un correcto diseño institucional. Las reglas deben ser claras, simples y consistentes, a efectos de disminuir los costos asociados a su aplicación, y asimismo, deben dar cuenta de una regulación coherente que posibilite la certeza necesaria para que los agentes económicos puedan sujetar su comportamiento a tales normas. Al respecto, ver: SunSTEIN (2006).

21 Correa et al. (2000) p. 400.

22 En relación a la reforma del procedimiento concursal, ya hemos señalado que ella se concretiza en la nueva Ley sobre Reorganización y Liquidación de Empresas y Personas. Dicha reforma distingue claramente procedimientos concursales de Reorganización y procedimientos concursales de Liquidación, con fuerte influencia del derecho norteamericano. Los procedimientos concursales de Reorganización (PcR) son dos: (i) Procedimiento concursal de Reorganización normal (PcRn), tratado en los artículos 54 y siguientes, y (ii) Procedimiento concursal de Reorganización Simplificado (PcRs), regulado en los artículos 102 y siguientes. El segundo se distingue del primero en cuanto a que el acuerdo que supone la Reorganización se obtiene extraconcurso, o sea de manera extrajudicial, debiendo posteriormente ser homologado ante el juez competente respectivo.

23 En este sentido, según hemos visto el modelo de litigación del neutral al riesgo se define como:

$P(W-M-C l+M)+(1-P)(W-M-C l) \geq W-M$

Es del caso que si el factor de riesgo del adverso al riesgo es $[\mathrm{r}(\mathrm{w})>\mathrm{O}]$, entonces, el factor respectivo, tendrá valor positivo, y deberá sumar aquella parte de la ecuación que representa la alternativa del sujeto de no ir a juicio. Por lo mismo, en este escenario, habrán menos juicios que en el supuesto (1), ya que tendrá mayor valor aquella parte de la ecuación que refiere la alternativa de no ir a juicio, según lo siguiente:

$P(W-M-C l+M)+(1-P)(W-M-C l) \geq W-M+\mathrm{r}(\mathrm{w})$

Por su parte, en el caso que del factor de riesgo del propenso al riesgo, el mismo tiene valor negativo $[\mathrm{r}(\mathrm{w})<0]$, y se deberá restar a aquella parte de la ecuación que representa la alternativa del sujeto de no ir a juicio. Por lo tanto, en este tercer escenario, habrá más juicios que en los escenarios (1) y (2) [3>2>1], ya que aquella parte de la ecuación que refiere la alternativa de no ir a juicio será menor, según lo siguiente:

$P(W-M-C l+M)+(1-P)(W-M-C l) \geq W-M-r(w)$ 
En este sentido, corresponde asociar al litigante con una definición determinada, que entendemos se corresponde con un litigante adverso al riesgo, toda vez que, constituye la forma más normal en que las personas se representan frente al ejercicio de una acción judicial ${ }^{24}$. En efecto, cabe señalar que la posición de neutralidad al riesgo es excepcional, ya que habitualmente las personas tenemos una posición sobre las posibles eventualidades de los cursos causales existentes y venideros. Por otro lado, la posición de propensión al riesgo no resulta deseable como modelo de política pública, toda vez que, refleja una preferencia asociada a personalidades muy específicas (jugadores de azar, alpinistas, etc.). De esta forma, en las explicaciones siguientes y en este trabajo, no nos extenderemos en el análisis de aquellos modelos de litigación que conducen a explicar el comportamiento de litigantes con neutralidad o propensión al riesgo, centrándonos en el litigante neutral al riesgo.

\section{LAS DEFICIENCIAS DE LA TESIS TRADICIONAL}

Hemos tenido la oportunidad de mencionar en el acápite anterior, en qué consisten a grandes rasgos los postulados de lo que hemos denominado tesis tradicional. Al respecto hemos identificado ciertas deficiencias de dicha posición -en su análisis tanto de la demanda de servicios de justicia, como de la oferta de tales servicios-, que las desarrollaremos con mayor detención en las dos secciones de este acápite, la primera dedicada a las deficiencias de la tesis tradicional en lo que se refiere a la demanda de servicios judiciales (3.1), y la segunda referida a las deficiencias de la oferta de servicios de justicia (3.2).

\subsection{Deficiencias de la teSis tradicional EN EL ANÁlisis REFERENTE A LA OFERTA DE} SERVICIOS JUDICIALES.

En primer lugar, abordaremos lo referente a las deficiencias de la tesis tradicional en sus postulados desde la perspectiva de la oferta.

Para comenzar, discutiremos la forma en que tales autores entienden el concepto de bien público:

(i) Lo primero que quisiéramos discutir es la tesis que descarta la existencia de un bien público en los servicios de justicia actuales, sobre la base de señalar que las externalidades no son internalizadas por los demandantes. Sin embargo, entendemos que ello es un error, toda vez que, exige a un bien público que las externalidades que produzca, sean internalizadas por los usuarios que consumen dicho bien, en circunstancias que por naturaleza el bien público al ser financiado por todos vía impositiva, permite al Estado ${ }^{25}$-esto es a quien ofrece el bien público, y a no a quienes lo demandan- internalizar sus externalidades ${ }^{26}$. Ello genera una inconsistencia que

\footnotetext{
${ }^{24}$ En este sentido Robert Cooter y Thomas Ulen: "Los economistas suponen que la mayoría de los individuos sienten aversión por el riesgo, pero algunos individuos son neutrales hacia el riesgo o, como los jugadores, los alpinistas y los corredores de carros de carrera, prefieren el riesgo" (COOTER y ULEN (2000) p. 81).

25 Olson (1965) p. 15.

26 GonzÁlez (2009) p. 5 y Benegas (1998)
} 
se produce cuando se pretende desestimar la existencia de un bien público en base a exigencias que no son propias del concepto de bien público. Evidentemente que de seguir ese predicamento, la administración de justicia no será considerada nunca un bien público, porque no se daría uno de sus supuestos requisitos, sin embargo, dicho supuesto requisito no es tal, por lo que la tesis tradicional equivoca su análisis al constatar un efecto -que los demandantes del bien público no internalizan sus externalidades- que no guarda relación con el concepto de bien público, y

(ii) Lo segundo que quisiéramos discutir con la tesis tradicional, es a nivel conceptual, lo que ellos entienden por bien público, toda vez que, descartan que estemos en presencia de un bien de dicha naturaleza, por el hecho de que: "no es verdad (...) que no exista rivalidad por acceder al aparato de justicia, o que no sea posible excluir a un nuevo consumidor". Sin embargo, no compartimos que: (ii.1) Exista rivalidad por acceder a la justicia, ya que, la oferta de justicia en nuestro país se sujeta a criterios orgánicos de inexcusabilidad, que en términos económicos, significa que se trata de un bien inagotable desde el punto de vista de su provisión, y por tanto, propiamente un bien público. Así, los agentes económicos conocen que cualquiera que sea la decisión de un litigante potencial (demandar o no demandar), ello no se traducirá en un desmedro en la elección óptima de su propia decisión (demandar o no demandar). Desde la teoría de juegos, no existe una alternativa predominante, que afecte la decisión de consumo de justicia relativa a dar inicio a un procedimiento judicial; (ii.2) También controvertimos que se pueda excluir a un nuevo consumidor en los servicios de oferta de justicia como los existentes en Chile; de hecho, ello se opone, con lo señalado anteriormente por los autores, en cuanto a que postulan que no existirían: "barreras aparentes de acceso como lo serían, por ejemplo, un sistema de precios". El problema se presenta, toda vez que, la inexistencia de tasas judiciales, sería una demostración de la inexistencia de barreras aparentes de acceso, ya que precisamente el establecimiento de dichas tasas es lo que constituiría el principal motivo para excluir a un consumidor del acceso a la justicia ${ }^{27}$. No obstante ello, los autores de la tesis tradicional proponen el establecimiento de tales tasas, lo que significaría, contraproducentemente con su posición, la generación de las barreras de acceso.

27 En este sentido, el establecimiento de tasas vendría a aumentar los costos del proceso respecto de la situación de litigación que hemos descrito anteriormente:

$$
P(W-M-C l+M)+(1-P)(W-M-C l) \geq W-M
$$

En efecto, si establecemos tasas judiciales tendríamos un nivel menor de litigación que en el óptimo anterior, constituyendo aquellas una barrera monetaria $(C l)$ no existente en el modelo indicado.

En la práctica las tasas judiciales lo que buscan es configurarse como filtros para el ejercicio de ciertas acciones, en términos tales que algunas demandas potenciales en ausencia de tasas pudieran presentarse, con el establecimiento de ellas dejan de ser factibles. La razón es la insuficiencia patrimonial a que hemos hecho referencia, en cuanto a que debido a la posibilidad de perder un proceso, para un litigante existirá un gasto efectivo, durante el curso del juicio que determine una renta neta negativa (W-M-Cl $<0$ ), y que por tanto, dicha situación impida el ejercicio de la acción. De esta forma, habrá acciones que no se presenten, no solamente por no poseer plausibilidad interna (lo que cumpliría el objetivo socialmente deseable del establecimiento de las tasas), sino que también, porque es posible la falta de financiamiento del litigante procesal durante el proceso, bajo la expectativa de resultar pernicioso (lo que no cumpliría con algún objetivo socialmente deseable, sino que generaría problemas de acceso de justicia). 
Hemos visto en el acápite anterior, que otra de las deficiencias en el análisis de la tesis tradicional recae en que para descartar la existencia de un bien público desde la perspectiva de la oferta, terminan equívocamente derivando una problemática relativa a la demanda de servicios de justicia ${ }^{28}$.

En último término, la búsqueda de un óptimo de oferta o provisión de servicios de justicia, según hemos visto, vendría dado por el cumplimiento de dos requisitos copulativos, a saber: (a) cuando todos están protegidos por el efecto disuasivo o represivo y (b) cuando el Estado subsidia la demanda en una magnitud equivalente a las externalidades positivas que genera quien recurre al sistema. Dichos supuestos óptimos, son inalcanzables desde un punto de vista regulatorio, y en lo que se refiere al primer supuesto mencionado, bastaría una nueva forma de entender la labor jurisdiccional para entender satisfecho dicho requisito, sin que se requiera entender la administración de justicia como un bien privado, veamos:

En lo que se refiere al requisito de producción de efectos disuasivos por parte del sistema de justicia, cabe indicar que no resulta posible concebir un requisito óptimo de provisión de justicia, exigiendo la generación de externalidades públicas positivas referentes a un sistema de precedentes, sobre el entendido que el único beneficio social identificable de un sistema de administración de justicia, es la producción de una uniforme interpretación de las normas, en términos que genere certezas jurídicas suficientes a favor de los sujetos destinatarios de dichas reglas. En un sistema judicial como el nuestro, dicho objetivo es un imposible en términos absolutos.

Son los mismos autores de la tesis tradicional quienes demuestran cuantitativamente que el sistema de justicia civil y comercial no genera tales externalidades. Ello no resulta difícil de constatar, considerando una de las reglas básicas de la definición de la labor de adjudicación, referida al artículo 3 del Código Civil ${ }^{29}$, en cuanto basa el ejercicio judicial en la resolución del caso concreto. Adicionalmente, el instituto que naturalmente está predispuesto por el legislador para producir externalidades públicas positivas o precedentes -la casación-, no solamente no cumple esa labor, sino que además, dogmáticamente es difícil que lo pueda alcanzar, en la medida que existe una delimitación normativa de sus reglas que conduzca a entender a dicho recurso desde la perspectiva del proceso, y no desde una perspectiva institucional ${ }^{30}$. Lo anterior, sumado a todas las dificultades propias del funcio-

\footnotetext{
${ }_{28} \mathrm{Al}$ respecto ver: pie de páginas 6 y 7.

29 En este sentido, he señalado que: "la posibilidad del juez de tomar una decisión que signifique otorgar incentivos e información a los individuos para que en casos futuros puedan ajustar su conducta a reglas claras y definidas en precedentes asentados en razón de criterios que incluyan razonamientos económicos, más que centrar su atención a las repercusiones, que puede tener la sentencia, en el bienestar de las partes, resulta completamente alejada de la manera en como Andrés Bello entendió la labor del juez, en cuanto a la extensión de sus resoluciones por cuanto, el artículo tercero inciso segundo de dicho cuerpo de leyes, establece que: "Las sentencias judiciales no tienen fuerza obligatoria sino respecto de las causas en que actualmente se pronunciaren" Carrasco (2012) p. 59.

30 En este sentido, si bien las Cortes de Casación poseen como finalidad la obtención de la nomofilaquia, entendida como la función de realizar la elección de la interpretación justa de la norma, ello puede alcanzar resultados diversos según el modo en el cual la Corte interpreta su propio rol. Así, si la Corte de Casación lo que pretende es poner en evidencia los errores de Derecho en que incurren los jueces de mérito en las decisiones precedentes sobre el caso concreto, entonces, la actividad interpretativa estará dirigida hacia el pasado, y pretenderá controlar el fundamento jurídico de una decisión ya tomada (rol de Casación desde el punto de vista del
} 
namiento de un sistema de casación, referentes a la prácticamente imposible diferenciación entre aspectos fácticos y jurídicos ${ }^{31}$, que evidentemente repercute en que aquello que debe ser susceptible de uniformidad (aspectos de Derecho), no se logre alcanzar.

Un escenario como el que se ha presentado, nos puede conducir a dos posibles respuestas: (i) La primera es aquella que sostiene la tesis tradicional, esto es, dada la incapacidad del sistema de administración de justicia para producir precedentes, entonces, no existe beneficio social identificable asociado a dicha oferta de servicios de justicia, y por tanto, todo gasto estatal que financie una labor puramente regresiva, debe ser repensada, y atribuir dichos gastos a quienes son usuarios del sistema. En otras palabras, enfrentar la crisis de la administración de justicia entendida como bien público - por incapacidad de obtención de beneficios identificables-, transformando la provisión de justicia en un bien privado; (ii) La segunda respuesta es la que queremos plantear sucintamente en este trabajo, aun cuando hemos hecho apología de la misma en otras oportunidades ${ }^{32}$. Al respecto, es posible modificar la forma de adjudicar justicia, entendiendo al Análisis Económico del Derecho normativo como pauta de interpretación general de las reglas del sistema jurídico ${ }^{33}$.

proceso); en cambio, si lo que busca es la individualización de la interpretación más justa de la norma aplicable al caso concreto entonces, la actividad interpretativa estará dirigida esencialmente hacia el futuro, y su objetivo principal es orientar la jurisprudencia sucesiva, esto es, generar un precedente para la correcta interpretación de la norma (rol de Casación desde el punto de vista institucional) (Ver: TARUffo (2006). Evidentemente los autores de la denominada tesis tradicional, ni siquiera tratan ni analizan el rol de la Corte Suprema como Corte de Casación. De haber analizado dicho trascedente tópico deberían haber concluido que la configuración del rol de Casación chileno está pensado desde el punto de vista del proceso, y por tanto, incapacitado para generar verdaderos precedentes.

31 Ver: TARuffo (2006) pp. 167-169 y Atria (2004). En el plano jurídico la distinción entre quaestio iurisy quaestio facti ha sido doblemente cuestionada. Por una parte, argumentando que la quaestio facti concierne a los hechos jurídicamente calificados y no a los hechos brutos; o si se quiere, que la premisa fáctica del silogismo no es un simple enunciado descriptivo de un acontecimiento sino el resultado de una operación judicial mediante la cual se califican unos hechos, y esa operación de calificación jurídica de los hechos tiene naturaleza normativa. Por otra parte, la distinción también se ha cuestionado argumentado que lo que cuenta como quaestio facti y lo que cuenta como quaestio iuris varía de sistema en sistema, y a veces de jurisdicción en jurisdicción, y que esa falta de unanimidad y caridad legal ilustra la dificultad de establecer una distinción a priori entre ambas (GASCÓN (2012) pp. 12 a 14).

32 Ver: Carrasco (2012) p. 380; Carrasco (2010); Núñez y Carrasco (2011) y Carrasco (2012).

33 Dicha modificación en la forma de adjudicar justicia necesariamente debe suponer la derogación del artículo 3 del Código Civil, toda vez que, tal norma consagra la base de la decisión judicial en atención al caso concreto. Por el contrario el Análisis Económico del Derecho se traduce en un juicio de valor, que busca evaluar los fines del ordenamiento jurídico, sobre la base de la eficiencia, en el entendido que las leyes son instrumentos para lograr metas sociales, tendiendo ellas a que siempre se opte por la decisión óptima desde una perspectiva económica. Debido a que un juez debe atribuir derechos en el marco de una contienda jurisdiccional, entonces debe propender aplicando la normativa, a una decisión que establezca aquel precedente que tienda a una asignación eficiente de los recursos. De esta manera el análisis económico del derecho normativo posee un evidente propósito prescriptivo. Así, en sus aplicaciones podemos encontrar la génesis de aquellas normas que conforman un conjunto jurídico inspirado en sus planteamientos. En este sentido, si según Arthur Kaufmann, toda legislación positiva presupone ciertos "principios generales del derecho" (Erick Wolf), “principios ético-jurídicos” (Larenz), "máximas de actuar justo" (Wieacker), que constituyen mandamientos fundamentales que orientan una determinada legislación, entonces, el análisis económico del derecho normativo se erige como uno de aquellos. Con ello, se transforma en un criterio de valoración constitutivo del fundamento del orden jurídico, sirviendo como exigencia de política legislativa de la normativa que se inspira en sus postulados. Asimismo, adquiere la calidad de instrumento de interpretación en casos dudosos, en el proceso de realización del derecho. Precisamente su 
Una aclaración previa, existen dos conceptos de Análisis Económico del Derecho: análisis Económico del Derecho positivo (AEDp) y Análisis Económico del Derecho normativo (AEDn). Cada uno de dichos conceptos responden a preguntas diferentes; así la pregunta: ¿De qué manera se afecta el comportamiento humano con la promulgación de una norma jurídica determinada?, va a producir una respuesta distinta a la pregunta: ¿Por qué resulta preferible un conjunto normativo que promueve la eficiencia, a otro que no? En efecto, la respuesta a la primera pregunta, supone que los sujetos actúan como entes que tienden a maximizar sus beneficios, de manera que considerarán la norma jurídica promulgada, como un precio implícito en la toma de sus decisiones ${ }^{34}$. Lo anterior constituye el objeto del análisis económico del derecho positivo. Por su parte, la respuesta a la segunda pregunta, busca generar un cambio normativo, sobre la base de una premisa de eficiencia en la asignación de los recursos. Ello supone, evidentemente, efectuar un juicio de valor acerca de la preeminencia de la eficiencia como criterio que debe orientar la legislación. Ello importa que se valore la eficiencia (como premisa que subyace a la ley), de mejor manera que otras premisas, como por ejemplo, la equidad. Lo anterior constituye el objeto del análisis económico normativo.

Considerando lo anterior, podemos describir ambas perspectivas señalando que: el análisis económico del derecho positivo, pone énfasis en la descripción y explicación del contenido de un conjunto normativo o de prácticas contractuales. Por su parte, el análisis económico del derecho normativo, tiene como tarea determinar cómo el ordenamiento jurídico puede ser utilizado para obtener determinar fines, tales como, la prevención de conductas indeseadas o ineficientes, desde la óptica de la economía ${ }^{35}$. En otras palabras, el análisis positivo, describe la situación tal cual es, en cambio, el examen normativa prescribe cómo debe ser dicha situación.

La novedad del análisis económico del derecho, se debe a que introduce la técnica económica moderna al estudio del derecho, pretendiendo dotar a este de eficiencia, y de una mirada consecuencialista, desde su perspectiva normativa ${ }^{36}$. La consecuencia de ello es el tránsito desde la intuición económica del problema jurídico, hacia el análisis económico del derecho ${ }^{37}$.

De esta forma, si un juez en el sistema continental de base romanista, tiene la posibilidad de optar por la eficiencia ${ }^{38}$ al valorar las consecuencias alternativas que puede generar

trascendencia es que sirve de pauta hermenéutica para descifrar el sentido y alcance de los pasajes oscuros y contradictorios de las normativas que en él se inspiran, las cuales pueden interpretarse de acuerdo a su formulación, todo de conformidad al artículo 24 del Código Civil.

34 Cooter y Ulen (2000) p. 14.

35 Paolantonio (2006) p. 207.

36 Paolantonio p. 202.

37 Así, hoy en día el análisis económico del derecho, si bien reconoce un origen impropio en dichas expresiones de sentido común, resulta forzoso concluir que sus inicios se encuentran vinculados con la economía moderna.

38 Una posición contraria sostiene Bentham ya que para él, un juez se debe comportar como un utilitarista de la regla, en virtud del cual, debe aplicar las leyes maximizadoras de bienestar emitidas por un legislador utilitario, aunque en un caso concreto, ello signifique no incrementar ni maximizar la felicidad, ello porque de acuerdo a la máxima de Bentham el juez siempre debe aplicar las leyes emitidas por el legislador. Desde este punto vista, e impidiendo que la judicatura se comporte como un utilitarista del acto, Bentham estaría cerrando la 
su decisión, entonces, tendría el ámbito de competencia suficiente para poder aplicar, en su tarea de resolución de escenarios de elección social, la perspectiva normativa del law and economics. Eso supondría que la labor de adjudicación se transformaría en la posibilidad del juez de tomar una decisión que signifique otorgar incentivos e información a los individuos para que en casos futuros puedan ajustar su conducta a reglas claras y definidas en precedentes asentados en razón de criterios que incluyan razonamientos económicos. La labor de jurisdiccional dejaría de centrar su atención a las repercusiones que puede tener la sentencia, exclusivamente en el bienestar de las partes.

Como podemos apreciar la obtención de un óptimo de oferta vinculado al requisito que el sistema de administración de justicia esté en condiciones de otorgar un efecto disuasivo general, produciendo externalidades públicas positivas, que justifiquen el gasto estatal por medio de recursos provenientes de rentas generales, solamente es posible de ser alcanzado, a través de un sistema jurisdiccional que considere la labor judicial de manera consecuencialista, mirando hacia el futuro, preocupándose de la generación de incentivos a los sujetos, y aplicando criterios de eficiencia en la resolución de los casos. De esa forma, seguiríamos entendiendo la justicia como bien público evitando su privatización, fundada esto último en la conclusión de la tesis tradicional, en cuanto a que el sistema de administración de justicia, no está capacitado para producir aquellas externalidades públicas positivas cuya ausencia justificaría entender la justicia como bien privado.

Para terminar el análisis de las deficiencias de la tesis tradicional en lo que se refiere a la perspectiva de la oferta, el segundo requisito de óptimo que se exige por los autores que sostienen la tesis tradicional refiere que el Estado debe estar en condiciones de subsidiar la demanda en una magnitud equivalente a las externalidades positivas que genera quien recurre al sistema. Esto es, debe encontrarse en condiciones de poder identificar ex ante las externalidades públicas positivas que una acción va a generar, a efectos de entregar un subsidio solamente a aquellas demandas que van a producir las mencionadas externalidades.

Resultan evidentes las dificultades regulatorias que surge de una propuesta como aquella. En primer lugar, una regulación como la señalada no toma en consideración los costos que impone ${ }^{39}$. En efecto, basta revisar el enunciado del segundo óptimo que indican los autores para darse cuenta que requieren de un proceso regulatorio complejo para ponerlo en práctica. Se necesitarán estudios cuantitativos de los resultados (léase, externalidades positivas) de las acciones judiciales que se inicien, no para una generalidad de acciones, sino que para cada acción específica porque el presupuesto del óptimo que se analiza, supone que solamente son subsidiadas aquellas acciones que generan precedentes, de esta forma, el esfuerzo regulatorio supondría establecer una agencia estatal que pueda calcular dicho subsidio. Al respecto se debe llamar la atención que los errores del proceso regulatorio, también imponen costos, de forma que cualquier agencia que tenga que asumir la tarea de

puerta a una aplicación inmediata de criterios de eficiencia donde el legislador no lo haya contemplado. Así: Moreso (1992) p. 360. Una posición contraria sostiene: Postema (1986) p. 430.

39 En este sentido, dando cuenta de la importancia de considerar los gastos que una regulación legal impone: STIGLER (1974) p. 58. 
calcular el subsidio deberá estar en condiciones de poder llevar a cabo en forma debida su tarea, de otra forma, será imposible generar un gasto público focalizado y no regresivo.

Basta hacer presente las consideraciones anteriores para que nos quede claro, que el aparato regulatorio no está en condiciones de establecer un sistema de cálculo de externalidades que justifique un subsidio como el que proponen los autores. Los costos de establecer un mecanismo que conduzca a dichos resultados, son claramente superiores a los beneficios que se pueden generar, máxime si consideramos que sin un cambio del paradigma en la forma de ejercer la función jurisdiccional, la búsqueda del precedente resulta imposible, según lo visto anteriormente en este acápite. En otras palabras, no solamente los costos regulatorios asociados a la búsqueda del segundo óptimo señalado son altísimos, y sujetos a una gran contingencia de error -lo que aumenta asimismo, los costos de la regulación-, sino que además, no se divisan beneficios sociales del establecimiento de una regulación como la indicada, sin que exista una modificación en la forma del ejercicio jurisdiccional.

Se entenderá fácilmente que un gasto social sin retribución de beneficio alguno, resulta más regresivo para las finanzas del Estado, que la inversión actual en administración de justicia sobre el discutible entendido de culpar a dicha oferta de servicios judiciales por no alcanzar ciertas externalidades que no se pueden obtener con la forma actual de comprender dicha oferta jurisdiccional.

En segundo lugar, la propuesta regulatoria de los óptimos de oferta de la tesis tradicional, es difícil de alcanzar, utilizando métodos que consideramos no sirven para determinar la extensión del óptimo que pretende conseguir ${ }^{40}$. En efecto, la propuesta supone el establecimiento de un subsidio al ejercicio de una acción, y que por tanto, debe ser aplicada al momento en que se inicia la acción o demanda, sin embargo, el subsidio se calcula en razón de los precedentes que dicha acción genera, esto es, el cálculo va a depender de un factor que se conocerá no al momento en que se inicia la demanda, sino que cuando la misma finalice con una sentencia, y que incluso deberá poseer un cualidad adicional -vinculado con la inexistencia de error por parte del adjudicador-, referido a que la sentencia deberá tener la virtud del producir disuasión o alguna externalidad pública positiva equivalente. En otras palabras, el criterio de óptimo, no determina correctamente la forma de cálculo del método con el que se pretende obtener dicho óptimo, toda vez que, no existirá corrección alguna si los antecedentes necesarios para calcular el subsidio no se conocen a la fecha en que el subsidio debe ser establecido.

Tales problemas en la detección de un óptimo vinculado con la oferta de servicios de justicia, se puede deber a una falta de entendimiento acerca de la naturaleza de una racional configuración de la administración de justicia. Para efectuar un tratamiento debido del problema, no debemos extrapolar simplemente recomendaciones teóricas del law and economics, sin considerar las particularidades del sistema en que tales recomendaciones pretenden implementarse. No se necesita entender la justicia como bien privado para alcanzar un óptimo de oferta de servicios judiciales. Por el contrario, basta cambiar la forma de entender la labor jurisdiccional aplicando los mismos recursos actualmente existentes, sin

\footnotetext{
40 Ineficiencias regulatorias similares, han sido puestas en evidencia en aspectos relativos al óptimo de ejecución o cumplimiento normativo o legal, ver: STIGLER (1974) pp. 55-67.
} 
necesidad de calcular subsidios ni externalidades, para alcanzar iguales o mejores eficiencias, e incluso -aunque suene un poco contraproducentemente- poder seguir entendiendo la administración de justicia como bien público.

\subsection{DEFICIENCIAS DE LA TESIS TRADICIONAL EN EL ANÁLISIS REFERENTE A LA DEMANDA}

\section{DE SERVICIOS JUDICIALES}

Corresponde que analicemos a continuación las deficiencias de la que hemos denominado como tesis tradicional en lo relativo al análisis que los autores que sostienen dicha posición realizan respecto de la demanda de servicios de administración de justicia.

Según hemos visto para los autores de lo que hemos denominado la tesis tradicional, la forma de afrontar los problemas de demanda de servicios de justicia, pasa por imponer a los litigantes parte de los costos sociales que impone el ejercicio de sus acciones. Dicho objetivo se logra por medio de tasas judiciales. En este sentido, los autores que hemos mencionado en este trabajo señalan que dichas tasas deben ser calculadas de la siguiente forma: "(...) cubrir el importe del servicio proporcionado menos el monto proporcional de las externalidades generadas" ${ }^{41}$.

Tal como hemos indicado en otra oportunidad ${ }^{42}$, tal solución resulta, empero, impracticable, en la medida que la tasa no se establezca de acuerdo a parámetros fácilmente calculables. Lo anterior, debido a que el cobro de tales tasas está sujeto a problemas regulatorios $^{43}$, por ejemplo: nuevamente - al igual que con el segundo requisito de óptimo de oferta de servicios judiciales- habría que dotar a la administración de justicia de personas calificadas capaces de valorar los elementos de la fórmula en que se concretice dicha solución; adicionalmente, no en todos los casos existirán externalidades positivas asociadas, y en aquellos casos, donde existan, se genera el problema de su valoración, sobre todo si consideramos que el beneficio de ellas se producirá a futuro, por medio de la certeza jurídica que el precedente proporcionará, de forma tal que no resulta posible valorar el elemento más importante de dicha solución en el caso particular, porque el único momento en que ello se podrá saber es cuando el proceso termine, e incluso más, con posterioridad a que ello ocurra, esto es, cuando el precedente genere efectos generales; finalmente, se deben considerar los mecanismos, procedimientos y costos del cobro de tales tasas, lo que determinará una regulación más costosa que aquella falla que se pretende salvaguardar con el proceso regulatorio.

41 Correa et al. (2000) p. 400.

42 Núñez y Carrasco (2014).

43 Uno de las características de un correcto marco regulatorio es lograr un consumo o demanda eficiente de los bienes en cuestión, en cuanto a que la tarificación de los servicios conduzca un comportamiento eficiente de los usuarios. Ver: Meller (2002); Joskow (1998). En este caso, dicho objetivo no se cumple, toda vez que, no es posible que los sujetos actúen eficientemente en sus decisiones de consumo, si aquella herramienta regulatoria que guiará o incentivará su decisión de acceso o no a justicia, no está asentada en bases o criterios claros (de hecho los requisitos asociados al cálculo de las tasas, no son evidentes ni aprehensibles por los sujetos destinatarios de las tasas, quienes no están en condiciones ex ante de valorar la cuantía de la misma, en circunstancias que el valor de dicha tasa, es la que definirá el ejercicio de su acción, según el modelo óptimo de acceso a justicia que hemos mencionado en el acápite 2 de este trabajo). 
El problema del establecimiento de tasas, refiere a un asunto largamente discutido en el law and economics ${ }^{44}$, y que pretende hacer coincidir el nivel de acciones judiciales con el nivel socialmente óptimo de demandas que debe recibir una administración de justicia. Sin embargo, lo novedoso en lo que hemos denominado tesis tradicional es la vinculación entre el monto de la tasa a aplicar y las externalidades positivas que del ejercicio de la acción se derivan, toda vez que, existen otros modelos que atienden más bien a hacer coincidir los costos privados y sociales de la litigación imponiendo una tasa a quien dé inicio a una demanda incorporando en la tasa los costos sociales y para terceros que genera el ejercicio de dicha demanda ${ }^{45}$, o bien, modelos en que la tasa esté asociada al beneficio que pudo haber obtenido el victimario demandado pernicioso ${ }^{46}$.

Si bien es cierto, todos dichos modelos generan evidentes problemas en el cálculo de la tasa a aplicar ${ }^{47}$-en el marco del objetivo de hacer coincidir el nivel de litigación privada con el nivel socialmente óptimo de ejercicio de acciones judiciales-, no es menos cierto que el cálculo que plantean en Chile los autores de la tesis tradicional está afecto a un problema mayor, porque uno de sus elementos según lo que hemos visto -obtención de ciertas externalidades- es difícil de lograr.

Una forma alternativa de alcanzar el deseable objetivo de hacer coincidir el interés privado $-\mathrm{y}$ correlativamente, los costos privados- con el interés social -y correlativamente, los costos sociales-, pasa por desarrollar herramientas procesales que considerando ambos aspectos del problema ${ }^{48}$, incentivando aquellas conductas que generen que dicha coincidencia se produzca con mayor probabilidad ${ }^{49}$. Ello supone por una parte, entender que es imposible establecer un sistema normativo que haga coincidir completamente los costos

\footnotetext{
44 Ver: SHAVELL (2004).

45 En este sentido, la posición del profesor Steven Shavell incorpora tales consideraciones al incluir en el cálculo del subsidio el factor del volumen óptimo de juicio. En este sentido, señala: "For the state to determine the optimal fee or subsidy, it must calculate the optimal volume of suit (determined by $h_{0}$, in the model), which requires it to know the incentive benefits of suit (and thus the function $p$ ) as well as the litigation costs. For the state to determine the optimal tax, to induce the correct level of care, the state need only ascertain litigation costs" SHAVELL (1999) p. 106.

46 Ver: POLINSKY y CHEN (1991).

47 En efecto, dichas dificultades quedan claras cuando se explican los elementos que se deben considerar para una regulación socialmente óptima a este respecto, Tales factores no son fácilmente administrables, como se puede apreciar en la siguiente cita: "The socially optimal outcome can be achieved under appropiate policy. (a) Victims can be induced to bring suit if and only if that is socially desirable, through use of a proper fee or subsidy: where there are too many suits, because the socially optimal threshold for bringing suit, $h_{0}$, exceeds victims'litigation costs, $c_{v}$, the state can set a fee for bringing suit equal to $h_{0},-c_{v}$, and where there are too few suits, because $h_{0}$, is less than $c_{v}$ the state can set a subsidy for bringing suit equal to $c_{v}-h_{0 ;}(b)$ In either case, injurers who are sued can be induced to exercise the socially desirable level of care by the state's imposing a tax equal to the public litigation costs, $c_{p}$, plus the victim's litigation costs $c_{v}$. SHAVELL (1999) p. 104.

48 La contribución en el sentido de valorar ambos aspectos del problema, proviene precisamente de Ronald Coase, quien en su publicación de 1960, en The Journal of Law and Economics de la Universidad de Chicago, titulada: "El problema del costo social", deja en claro que toda decisión jurídica de asignación de derechos significa un problema dual, ya que al beneficiar a A, se está perjudicando a B, frente a lo cual, y con miras a evitar el mal mayor, debe decidirse si se permite que A dañe a B, o bien que B dañe a A, ver: COASE (1992) p. 3.

49 Un análisis similar es el que expresa Steven Shavell, cuando analiza la necesidad de establecer una regulación para incentivar la entrega de información en el proceso, con fines de alcanzar una correcta determinación de hechos -y con ello, hacer coincidir objetivos sociales e intereses privados-, ver: SHAVELL (2004) p. 456.
} 
privados y sociales. Por otra parte, se debe entender que la existencia de una tasa de gasto regresivo se debe tolerar, máxime si el proceso está conformado precisamente para convivir con tasas de errores, a las que no debe temerse, ni pretender su eliminación total, ya que con ello estamos imponiendo una mayor cantidad de costos al sistema.

\section{CONCLUSIONES}

De esta forma, las conclusiones que podemos derivar de este trabajo son las siguientes:

1. Hemos denominado como tesis tradicional a aquellos autores nacionales que sostienen que los servicios de justicia (problema desde la perspectiva de la oferta) prestados por los tribunales que dependen del Poder Judicial, y que por tanto, son financiados con cargo a rentas generales, no deben constituir bienes públicos, sino que esencialmente privados, si es que constatamos que no permiten alcanzar externalidades positivas públicas.

2. Para tales autores, el problema desde la perspectiva de la oferta pasa por establecer óptimos que supongan: (a) la necesidad que la administración de justicia genere efectos disuasivos o represivos y (b) que el Estado subsidie la demanda en una magnitud equivalente a las externalidades positivas que genera quien recurre al sistema.

3. Sin embargo, tales objetivos son inalcanzables, en este sentido la obtención de externalidades públicas positivas (precedentes), es un imposible con la actual forma de entender el ejercicio jurisdiccional. Para que ello sea susceptible de ser alcanzado, cabe señalar que no se necesita entender la justicia como un bien privado para solucionar las problemáticas detectadas por tales autores, sino que basta establecer criterios de eficiencia como factores que justifiquen la decisión jurisdiccional (aplicando por tanto, análisis económico del derecho normativo).

4. En lo que se refiere al otro requisito de óptimo de oferta, relativo a la existencia de un subsidio equivalente al monto de las externalidades positivas que genera la deducción de una nueva demanda, cabe indicar que dicho criterio está afecto a problemas regulatorios que imposibilitan su aplicación.

5. Mismas dificultades existen respecto de la posición de la tesis tradicional en lo que se refiere a las problemáticas de la demanda de servicios judiciales. Para tales autores, el establecimiento de tasas judiciales es el medio para inducir que los costos privados que se generan por la litigación tiendan a coincidir con los costos sociales que produce el inicio de una demanda. Dicha tasa debe ser calculada de acuerdo al importe del servicio proporcionado menos el monto proporcional de las externalidades generadas.

6. Tal solución, no solamente es compleja de ser implementada desde un punto de vista regulatorio, sino que además, pone el acento en aspectos no considerados habitualmente por los exponentes del law and economics para calcular las tasas judiciales.

7. Todo ello, conduce a controvertir la conclusión de dicha posición en cuanto a entender la justicia como un bien privado. 


\section{BIBLIOGRAFÍA CITADA}

Atria, Fernando (2004): "Proceso Civil", Revista de Derecho Universidad Adolfo Ibáñez, N 2: pp. 249-353.

BENEGAS, Alberto (1998): "Bienes públicos, externalidades y los free-riders: el argumento reconsiderado”, Revista Estudios Públicos, N 71: pp. 204-218.

Carrasco, Nicolás (2010): "La contracautela: Una mirada desde el análisis económico del derecho procesal”, Revista de Derecho y Humanidades, vol. 2, Nº 16: pp. 171-185.

Carrasco, Nicolás (2012): "Análisis económico del derecho de la tutela cautelar en la legislación civil en Chile", Revista de Derecho, Escuela de Postgrado, Universidad de Chile, $\mathrm{N}^{\circ}$ 2: pp. 93-120.

Carrasco, Nicolás (2012): Análisis Económico de las Medidas Cautelares Civiles (Santiago, Editorial AbeledoPerrot).

CoAse, Ronald (1992): "El problema del Costo Social”. Revista de Estudios Públicos, № 45: pp. 81-134.

CoOter, Robert y Ulen, Thomas (2000): Derecho y Economía (Ciudad de México, Fondo de Cultura Económica).

Correa, Jorge, Peña, Carlos y Vargas, Juan Enrique (2000): “¿Es la justicia un bien público?”, Revista Perspectivas, Departamento de Ingeniería Industrial, Universidad de Chile, vol. 3, N²: pp. 389-409.

Gascón Abellán, Mariana (2012): Cuestiones probatorias (Bogotá, Universidad Externado de Colombia).

GonZÁlez, Isaí (2009): “Bienes públicos: una aproximación al debate”. Encrucijada, México, $\mathrm{N}^{\circ} 3$ : pp. 1-16.

Huerta, José Miguel (2012): "Reglas de costas y sanciones pecuniarias: un análisis económico para la reforma procesal civil en Chile" Disponible en: <http://www.sistemasjudiciales.org/content/jud/archivos/notaarchivos/355.pdf>

Hughes, James y Snyder, Edward (1995): "Litigation and Settlement under the English and American Rules: Theory and Evidence", Journal of Law and Economics, vol. 38, $\mathrm{N}^{\circ}$ 1: pp. 225-250.

Joskow, Paul (1998): "Regulatory priorities for infrastructure sector reform in development", Annual World Bank Conference on Development, World Bank.

Meller, Patricio (2002): "Regulación y competencia en los Servicios de Utilidad Pública. Una Introducción general”, Revista Perspectivas, Departamento de Ingeniería Industrial, Universidad de Chile, vol. 6, No 1: pp. 3-10.

MerY, Rafael (2006): “Una aproximación Teórica y Empírica a la Litigación Civil en Chile” Disponible en: <http://works.bepress.com/rafael_mery_nieto/1>

Moreso, José Juan (1992): La teoría del Derecho de Bentham (Barcelona, Editorial PPU).

NúNÉz, Raúl y CARrasco, Nicolás (2011): "La quiebra sin bienes: Una aproximación desde el análisis económico del derecho", Ius et Praxis, año 17, Nº 1: pp. 139-176.

Núñez, Raúl y Carrasco, Nicolás (2014): “Conceptos generales del Análisis Económico del Derecho Concursal”, en NúnEEZ, Raúl (Editor) y CARRASCO, Nicolás (coordinador), 
Presente y Futuro del Derecho Procesal Concursal Chileno. Una revisión desde el Análisis Económico del Derecho (Santiago, Editorial LegalPublishing).

Olson, Mancur (1965): “The logic of collective action”, Harvard Economic Studies, No 124.

Paolantonio, Martín (2006): "El análisis económico del derecho y la estructura societaria”, en Kluger, Viviana (coord), Análisis Económico del Derecho (Buenos Aires, Editorial Heliasta)

Polinsky, Mitchell y Chen, Yeon-Koo (1991): "Decoupling liability: Optimal incentives for care and litigation", Rand Journal of Economics, vol. 44, $\mathrm{N}^{\circ} 4$ : pp. 562-570.

Postema, Gerald (1986): Bentham and the Common Law Tradition (Clarendon Press, Oxford University Press).

Stigler, George (1974): “The Optimum Enforcement of Laws”. Disponible en: <http:// www.nber.org/chapters/c3626>

Shavell, Steven (1999): "The Level of Litigation: Private Versus Social Optimality of Suit and of Settlement", International Review of Law and Economics: pp. 99-115.

SHAVELl, Steven (2004): Foundation of Economics Analysis of Law (Harvard University Press)

Sunstein, Cass (2006): “The Virtues of Simplicity”, Yale Law Journal PocketPart, vol. 116, $\mathrm{N}^{\circ}$ 70: pp. 70-76.

TARUfFo, Michele (2006): El vértice ambiguo: Ensayos sobre la casación civil (Madrid, Editorial Palestra).

Vargas, Juan Enrique, Peña, Carlos y Correa, Jorge. (2001): "El rol del Estado y el Mercado en la Justicia”, Cuadernos de Análisis Jurídico, Serie Seminarios, Universidad Diego Portales, vol. 42: pp. 1-29. 
